\title{
The Effect of Shift Rotation on Variations of Cortisol, Fatigue and Sleep in Sound Engineers
}

\author{
Katia VANGELOVA ${ }^{1}$ \\ ${ }^{1}$ National Center of Public Health Protection, 15 Akad Ivan Geshov Boul., 1431 Sofia, Bulgaria \\ Received February 22, 2008 and accepted May 16, 2008
}

\begin{abstract}
The aim was to study the effect of rotation direction on the time-of-day variations of cortisol, fatigue and sleep in sound engineers broadcasting in a life show. The salivary cortisol and ratings of stress, sleepiness and fatigue were followed at three hour intervals in 25 sound engineers: 13 working very fast forward-rotating shifts and 12 working very fast backward-rotating shifts. Cortisol was assessed with radioimmunological kits. The participants reported for stress symptoms and filled sleep diary. Cortisol retained the typical diurnal pattern. The rotation direction interacted with the shift significantly and as a result higher cortisol values during the morning and night shifts in the backward rotating group were found as well as worse quality of sleep. Higher salivary cortisol during morning and night shifts and worse quality of sleep in engineers working very fast backward-rotating shifts may be an indication for insufficient recovery.
\end{abstract}

Key words: Field study, Discontinuous shift work, Rotation conditions, Stress, Insufficient recovery

Shift work is related to higher risk of sleep disturbances, fatigue and ill health, particularly gastrointestinal, metabolic and cardiovascular diseases ${ }^{1,2)}$. The rapid forward-rotating shift systems are considered to cause less problems and to give faster recovery in relation to both sleep and social activities ${ }^{3,4)}$. In contrast, the fast backward-rotating shift systems including quick returns are associated with poorer physical and psychological wellbeing and higher level of fatigue ${ }^{5)}$. Boquet et al. $^{6}$ ) found no significant effect of rotation direction for cortisol and melatonin in two-week experiment with counterclockwise versus clockwise rotation.

Cortisol, a reliable indicator of stress, displays pronounced variation across the time-of-day with high levels in the morning and low in the evening. Stress may alter the secretion levels and circadian pattern of the hormone. It is known that long term increase of circulating cortisol or changes in the circadian rhythm of the hormone increase the risk of metabolic and cardiovascular diseases, depression, etc. ${ }^{7)}$.

In Bulgaria some sound engineers, broadcasting in a life show work very fast rotating shifts. Eight years ago a forward-rotating shift system was proposed and implemented, but a part of the engineers wished to continue to work backward rotating shifts and their preferences were taken in account.

The aim was to study the effect of rotation direction on the time-of-day variations of cortisol, fatigue and sleep in sound engineers.

Twenty five sound engineers, broadcasting in a life show, with a job task to control sound parameters and quality of the signal through auditory and visual signals from the indicator appliances were studied. Their duties also included recording of performances, developing products, finding eventual break down in appliances, organizing connections, etc. They were working very fast rotating shifts, the start and end of the shifts were the same for all the studied subjects. The morning shift (M) started at 05:30 and continued to 13:30, the afternoon one (A) was from 11:30 to 19:30, the evening shift (E) from 17:30 to 24:30, night shift (N) from 24:00 to 06:00. The sound engineers were selected by random. They were divided in two groups on the base of direction of shifts rotation, as follows: forward rotating group (M A E R N R) - 13 sound engineers ( 4 men and 9 women) aged $45.1 \pm 7.3 \mathrm{yr}$ with length of service $21.4 \pm 11.3$ and backward rotating group (E A M N R R) - 12 sound engineers (4 men and 8 women) aged $51.7 \pm 6.0 \mathrm{yr}$ with length of service $27.4 \pm 8.7$. Informed consent was signed by all the subjects. The work was approved by the ethical 
committee of the National Center of Public Health Protection.

The concentration of saliva cortisol was followed on three hour intervals at the following time points: morning shift -06:00, 09:00 and 12:00; afternoon shift -12:00, 15:00 and 18:00; evening shift $-18: 00,21: 00$ and 24:00 and night shift $-24: 00,03: 00$ and 06:00. The participants were instructed to avoid food and liquids for $30 \mathrm{~min}$ before these time points. The cortisol samples were analyzed by radioimmunoassay using Spectria $\left({ }^{125} \mathrm{I}\right)$ radioimmunoassay kit (Orion Diagnostica, Finland). The withinassay $\mathrm{CV}$ ranged 0.8 to $5.2 \%$ and the between-assay $\mathrm{CV}$ never exceeded $8 \%$.

At the above time points the participants made ratings concerning stress, sleepiness and fatigue in a diary. The stress scale included nine response categories ${ }^{8)}$, five included verbal anchors: 1 -very low stress (very calm and relaxed), 3 -low stress, 5 -neither low nor high stress, 7 -high stress (high tension and pressure), 9 -very high stress. The participants also rated their sleepiness during the shifts using Karolinska Sleepiness Scale (KSS) (1 very alert, 3 -alert, 5 -neither alert nor sleepy, 7 -sleepy, 9 -very sleepy, fighting sleep, effort to stay awake), validated against physiological and behavioral measures ${ }^{9)}$. Also during the shifts they rated on the feeling of fatigue by 9 point scale ( 1 -very fresh, 3 -fresh, 5 -neither fresh nor tired, 7 -tired, 9 -very tired). The Chrobach's $\alpha$ for the scales was 0.78 .

At the end of every shift, the participants reported how their day had been in respect to different symptoms of stress. The questions were pointed towards feeling of tension, irritation, exhaustion, time pressure, difficulties in concentration (1 -not at all, 5 -to a large extent) and whether the workload was high and the rest not enough (1 -definitely enough, 5 -far from enough). The Chrobach's $\alpha$ for the scales was 0.66 .

The participants filled in the Karolinska Sleep Diary $(\mathrm{KSD})^{10)}$. The ratings were made by 5 -point scale $(1$ poor sleep, 5 -no problems with sleep). A sleep quality index (SQI) was formed of questions "restless sleep", "ease of falling asleep", "sleep quality" and "sleep throughout". The Chrobach's $\alpha$ for the used scales was 0.70 .

The coffee consumption and smoking were followed, too. Also the time between awakening and cortisol sampling was taken in account.

The statistical analyses were carried out with SPSS. Kolmogorov-Smirnov test was used to study the distribution of salivary cortisol and showed normal distribution $(p>0.05)$. The effect of two within-subject factors: shift (morning, afternoon, evening, night shift) and time-of-day (beginning, middle, end of the shift) and between-subjects effect of rotation direction (forward/backward) of salivary

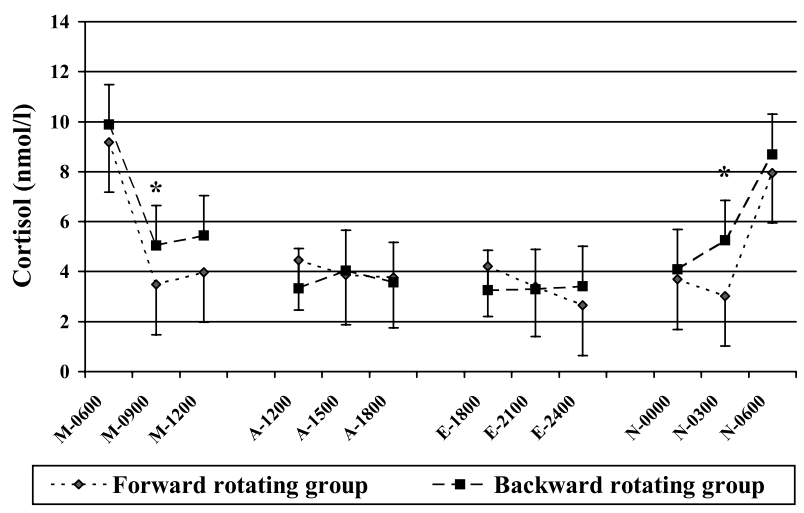

Fig. 1. Time-of-day variations of salivary cortisol (mean \pm SD) in sound engineers working fast forward- and backward-rotating shifts $(* p<0.05$ of $t$-tests).

cortisol and ratings of stress, sleepiness and fatigue were estimated using general linear model module for repeated measurements. The age of the studied subjects was included as co-variate in the statistical models. When the between-subjects effects showed significant interaction a comparison at each time point was made using $t$-tests. Daily symptoms of stress were followed with the general linear model module with a within-subject factor the shift, between-subjects effect - rotation direction. The effect of rotation direction on sleep was studied with oneway ANOVA.

Cortisol retained the typical diurnal pattern with high values in the early morning and lower secretion during the day (Fig. 1). The effect of rotation direction on cortisol secretion did not reach significance $\left(\mathrm{F}_{(1,22)}=4.004\right.$, $p=0.058$ ), but the effect of the shift was highly significant $\left(\mathrm{F}_{(3,66)}=24.357, p=0.001\right)$ with higher cortisol secretion during morning and night shifts. Significant interaction of rotation direction with the shift was found $\left(\mathrm{F}_{(3,66)}=6.046, p=0.001\right)$, higher cortisol values were found during morning and night shift in the backward rotating group. The effect of time-of day was also significant $\left(\mathrm{F}_{(2,44)}=15.175, p=0.001\right)$, as well as its interaction with rotation direction $\left(\mathrm{F}_{(3,44)}=5.086, p=0.01\right)$. The $t$-tests of each time point showed significantly higher cortisol values $(p<0.05)$ at 09:00 during the morning and at 03:00 during the night shifts in backward rotating group.

Stress ratings increased significantly with the time-ofday $\left(\mathrm{F}_{(2,44)}=10.456, p=0.001\right)$ in both groups (Fig. 2), reaching the highest level at the end of the shifts. Sleepiness differed significantly with the shift $\left(\mathrm{F}_{(3,66)}=17.833, p=0.001\right)$ with the highest scores in the night shift, followed by the evening ones. Sleepiness showed and effect of the time-of-day $\left(\mathrm{F}_{(2,44)}=15.813\right.$, $p=0.001$ ) and increased in both groups with advancing the shifts. Fatigue differed significantly with the shift $\left(\mathrm{F}_{(3,66)}=4.199, p=0.009\right)$ with the highest scores during the 

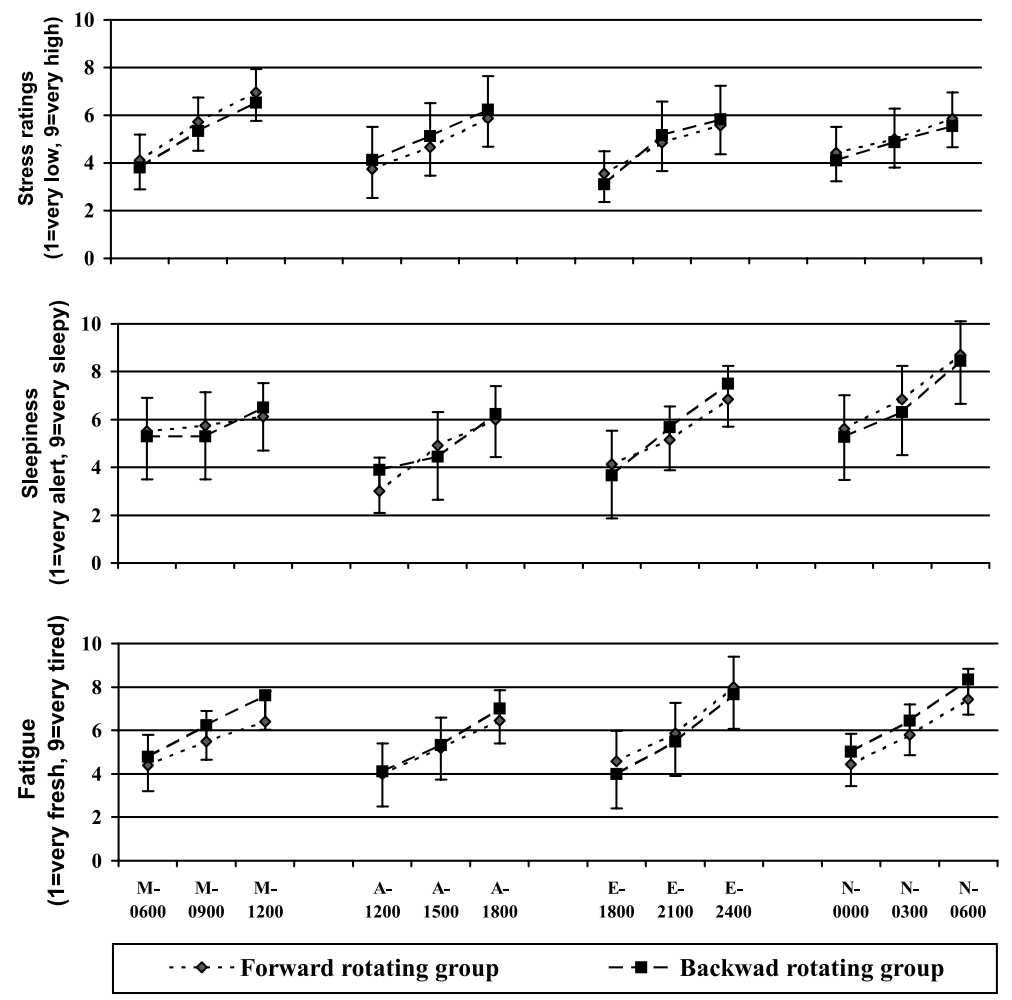

Fig. 2. Time-of-day variations of stress, sleepiness and fatigue scores (mean \pm SD) in sound engineers working fast forward- and backward-rotating shifts.

Table 1. Stress symptoms at the end of the shifts ${ }^{\ni}$, sleep duration and sleep $\operatorname{scores}^{5}$ in forward and backward rotating group of sound engineers broadcasting in a life show

\begin{tabular}{lcc}
\hline Indices / Groups & $\begin{array}{c}\text { Forward rotating } \\
\text { group (mean } \pm \text { SD) }\end{array}$ & $\begin{array}{c}\text { Backward rotating } \\
\text { group (mean } \pm \text { SD) }\end{array}$ \\
\hline Tense $^{\ni}$ (1=not at all, 5=to a large extent) & $3.67 \pm 1.17$ & $3.72 \pm 0.91$ \\
Irritated $^{\ni}$ (1=not at all, 5=to a large extent) & $3.21 \pm 0.88$ & $3.45 \pm 0.94$ \\
Exhausted $^{\ni}$ (1=not at all, 5=to a large extent) & $4.15 \pm 0.71$ & $4.06 \pm 0.89$ \\
High work load $^{\ni}$ (1=not at all, 5=to a large extent) & $4.17 \pm 0.57$ & $3.85 \pm 0.79$ \\
Rests $^{\ni}$ (1=definitely enough, 5=far from enough) & $3.79 \pm 1.04$ & $4.18 \pm 1.18$ \\
Sleep duration (h) $_{\text {Sleep quality index }}^{5}$ (1=poor sleep, 5=no problems with sleep) & $7.94 \pm 1.72$ & $7.82 \pm 0.88$ \\
Sufficient sleep $^{5}$ (1=poor sleep, 5=no problems with sleep) & $3.29 \pm 0.88$ & $2.71 \pm 0.62^{*}$ \\
Sleep fragmentation $^{5}$ (1=poor sleep, 5=no problems with sleep) & $3.68 \pm 0.75$ & $3.29 \pm 0.77$ \\
Early awakening $^{5}$ (1=poor sleep, 5=no problems with sleep) & $3.61 \pm 1.26$ & $2.94 \pm 0.83$ \\
\hline
\end{tabular}

$* \mathrm{~F}_{(1,29)}=4,514, p<0.05$.

night shifts. Fatigue increased with advancing the shifts in all the shifts in both groups (the time-of-day effect was $\left.\mathrm{F}_{(2,44)}=21.056, p=0.0001\right)$.

Our data showed no significant differences between the two groups for the reported stress symptoms (Table 1). The sound engineers felt rather tense and irritated and very exhausted at the end of the shifts. Both groups found also that the work load was high and the rests during work not adequate.

The reported sleep duration did not differ between the two groups (Table 1). The quality of sleep was worse in backward-rotating group in comparison to forward-rotating one. The backward rotating group also showed greater insufficiency of sleep, sleep fragmentation and early awakening, but the differences between the groups did not reach significance.

The age of the studied subjects did not show interaction with the studied indices. The coffee consumption and smoking showed no differences between the groups. Also no significant differences in terms of the time 
between awakening and cortisol sampling were found between the groups.

Considering cortisol and stress self ratings our data showed moderate stress levels with the studied sound engineers. The backward rotation was associated with higher cortisol values during morning and night shifts and worse sleep quality compared to forward one. Most researchers suggest that forward rotation produces less disruption of the circadian rhythm than backward one ${ }^{6,11)}$. There are data for lower amplitude and delay in the acrophase of some circadian indicators as oral temperature $^{6}$. Our data showed higher salivary cortisol during morning and night shifts in backward rotating group in comparison to forward rotating one. The work places and job task of the two groups did not differ. No significant differences between the two groups in terms of work load, time pressure, etc. were observed. The groups did not differ in terms of gender (male/female ratio). The age of the studied subjects was included in the statistical model, but did not show effect on cortisol values. The coffee consumption and smoking showed no differences between the groups, as well as the time between awakening and cortisol sampling. Thus following the salivary cortisol our data indicate that fast backward-rotating shifts contribute to higher physiological stress.

Barton and Folkard ${ }^{5)}$ found more health and well-being problems only when the backward-rotating shift system involved quick returns, with less than $8 \mathrm{~h}$ between the shifts. Our data showed significantly higher cortisol values in morning and night shifts with the studied sound engineers working backward-rotating shifts even when the rest period between the shifts was more than $10 \mathrm{~h}$. Also, the quality of sleep was worse in engineers working backward-rotating shifts. These data are in accordance with earlier findings ${ }^{3,13)}$. The disturbed sleep is considered as a common pathway connecting night shift work with adverse health effects, and the medium in between is the insufficient recovery ${ }^{14)}$. The shift schedules vary considerably in the time they offer for recovery. The forward rotation offers an easier change for the human circadian system from early to later shifts and more time for recovery, while the backward rotation -less time for recovery, and as our data show contributes to higher physiological stress.

In conclusion higher cortisol during morning and night shifts, higher fatigue and worse quality of sleep in sound engineers broadcasting in a life show on fast backwardrotating shifts may be an indication of insufficient recovery.

\section{References}

1) Åkerstedt T (2003) Shiftwork and disturbed sleep/wakefulness. Occup Med 53, 89-94.

2) Costa G (2003) Shiftwork and occupational medicine: an overview. Occup Med 53, 83-8.

3) Tucker P, Smith L, Macdonald I, Folkard S (2000) Effects of direction of rotation in continuous and discontinuos 8 hour shift systems. Occup Environ Med 57, 678-84.

4) Härmä M, Hakola T, Kandolin I, Sallinen M, Vikkala J, Bonnefonde A, Mutanen P (2006) A controlled intervention study on the effects of a very rapidly forward rotating shift system on sleep-wakefulness and wellbeing among young and elderly shift workers. Int $\mathbf{J}$ Psychophysiol 59, 70-9.

5) Barton J, Folkard S (1993) Advancing versus delaying shift systems. Ergonomics 36, 59-63.

6) Bouquet A, Cruz C, Nesthus T, Petwiler C, Knecht W, Holcomb K (2004) Clockwise and counterclockwise rotating shifts: effects on temperature and neuroendocrine measures. Aviat Space Environ Med 75, 898-904.

7) Chrousos GP (2000) The role of stress and the hypothalamic-pituitary-adrenal axis in the pathogenesis of metabolic syndrome: neuroendocrine and target tissuerelated causes. Int J Obes 24 (Suppl. 2), S50-5.

8) Dahlgen A, Kecklund G, Åkerstedt T (2005) Different levels of stress related stress and the effects on sleep, fatigue and cortisol. Scand J Work Environ Health 31, 277-85.

9) Åkerstedt T, Gillbert M (1990) Subjective and objective sleepiness in active individual. Int J Neurosci 52, 29-37.

10) Åkerstedt $T$, Hume $K$, Minors D, Waterhouse J (1994) The subjective meaning of good sleep- a interindividual approach using the Karolinska Sleep Diary. Percept Mot Skills 79, 287-96.

11) Boquet A, Cruz C, Nesthus T, Petwiler C, Knecht W, Holcomb K (2004) Clockwise and counterclockwise rotating shifts: effects on temperature and neuroendocrine measures. Aviat Space Environ Med 75, 898-904.

12) Sallinen $M$, Härmä $M$, Mutanen $P$, Ranta $R$, Virkkala J, Müller K (2005) Sleepiness in various shift combinations of irregular shift systems. Ind Health 43, 114-22.

13) Åkerstedt $T$ (1995) Work hours, sleepiness and underlying mechanisms. J Sleep Res 4 (Suppl. 2), 15-22.

14) Härmä M (2006) Workhours in relation to work stress, recovery and health. Scand J Work Environ Health 32, 502-14. 